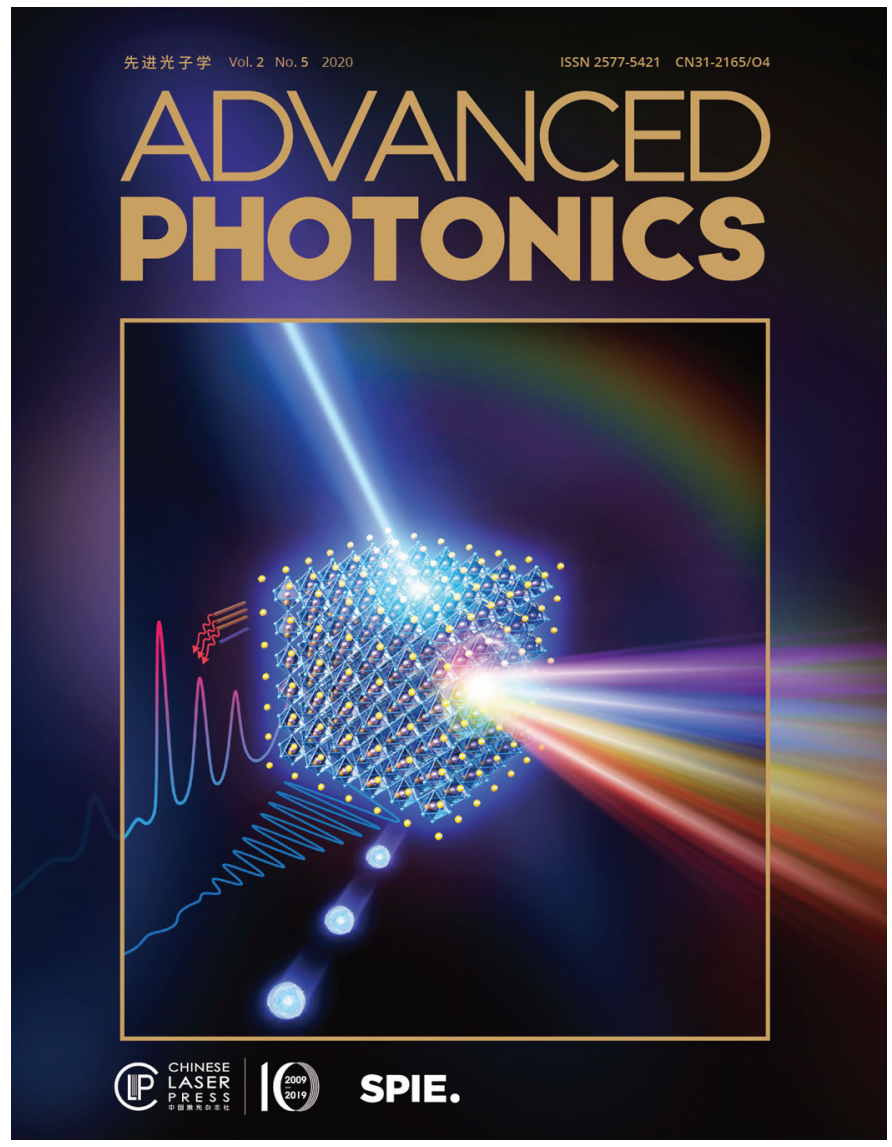

\title{
About the cover: Advanced Photonics Volume 2, Issue 5
}

The image on the cover for Advanced Photonics Volume 2 Issue 5 illustrates the collective optical properties of semiconductor perovskite nanocrystals under the excitation of upper blue laser ray.

The cubic perovskite nanocrystals exhibit amplified spontaneous emission (ASE) tunable from near UV to near infrared on the right side. Three sequential bright dots and decaying sinusoidal wave on the lower side represent single photon emission and quantum coherence in perovskite nanocrystals, respectively.
Meanwhile, the discrete energy levels and photoluminescence spectrum on the left side correspond to the fine structure of perovskite nanocrystals.

The image is based on the research presented in the review by Zengle Cao, Fengrui Hu, Chunfeng Zhang, Shining N. Zhu, Min Xiao, Xiaoyong Wang: "Optical studies of semiconductor perovskite nanocrystals for classical optoelectronic applications and quantum information technologies: a review," Adv. Photon. 2(5), 054001, doi: 10.1117/1.AP.2.5.054001. 